\title{
Long-term mental health support systems in the aftermath of disasters in Japan - Statistical data of the Miyagi Disaster Mental Health Care Center
}

Naru Fukuchi ( $\sim$ narufuku@za.cyberhome.ne.jp)

Miyagi Disaster Mental Health Care Center https://orcid.org/0000-0001-9856-1751

Shusaku Chiba

Tohoku University Graduate School of Education Faculty of Education: Tohoku Daigaku Daigakuin Kyoikugaku Kenkyuka Kyoiku Gakubu

\section{Research}

Keywords: mental health, earthquake, tsunami, Japan, post-disaster mental health service

Posted Date: December 3rd, 2020

DOI: https://doi.org/10.21203/rs.3.rs-119055/v1

License: () (7) This work is licensed under a Creative Commons Attribution 4.0 International License. Read Full License 


\section{Abstract \\ Background}

A long-term mental health support system for the community is sometimes needed following massive natural disasters. Although the Disaster Mental Health Care Center (DMHCC) was established as a long-term mental health care center in Japan, its exact role and functioning are unclear. The Great East Japan Earthquake and Tsunami (GEJE) of 2011 affected thousands of residents. The Miyagi DMHCC was established in Miyagi prefecture in response to the GEJE and supported residents and communities as a long-term mental health care center.

\section{Methods}

The main purpose of this study was to clarify which population is psychologically at high risk and which methods are useful for residents' mental health in each phase. The study used data collected by the Miyagi DMHCC that included personal information of residents who were supported by the center from 2013 to 2018. Chi-square tests of independence were conducted on the annual number of individuals supported by the center, sex, and the number of support methods used by the center according to years. A one-way analysis of variance was conducted on the annual mean age, followed by a post-hoc comparison of the functioning of the center.

\section{Results}

The number of residents who needed mental health support dramatically increased in Miyagi prefecture after the 2011 disaster. The Miyagi DMHCC supported 6,850 individuals who sought mental health services, which accounted for $22.9 \%$ of all cases reported to the health services between 2012 and 2017 . Based on the results, in the first few years, the elderly residents who lived alone were declared as high-risk individuals by the health survey and supported through home visits. Several years later, as younger people started to seek mental health support by themselves, they underwent counseling at the Miyagi DMHCC.

\section{Conclusion}

Residents who need mental health support might change depending on recovery phases. Long-term mental health care centers should observe community recovery and provide appropriate support. We discuss the implications of this result and future research directions.

\section{Introduction}

\section{The mental health needs following natural disasters depending on recovery phases}

The psychological needs of a community change with each recovery stage in the aftermath of large-scale natural disasters. McFarlane indicated that medical services tend to have priority in the rescue phase, whereas mental health services are highly demanded in the subsequent phases [1]. Although only a few residents need psychological support in the immediate aftermath of a disaster, mental health support teams need to make preliminary assessments of the resident's potential long term needs.

It has been reported that individuals do not seek help for their mental disorders on a voluntary basis. For example, Houston reported that social support for residents with probable post-traumatic stress disorder and depression was low, and these residents did not positively use mental health resources after the catastrophic 2011 Joplin tornado [2]. Similarly, Brown reported that several residents were at risk of suicide after observing the care provided by mental health providers in community settings (client's homes, schools, churches, and mobile units) in the aftermath of a series of hurricanes in Florida [3]. These experiences demonstrate that a team of well-trained providers who can screen for individuals at risk are essential and that active outreach based on screening is necessary.

In addition, North reported that a long-term mental health support system is needed to support residents with vulnerabilities and reconstruct a communitybased mental health system [4]. Significant adult mental health needs exist after 6 months and 18 months of the disaster having taken place [2]. Long-term programs aimed at monitoring, assessment, and outreach are needed for an extended period after a major disaster. Although the importance of long-term mental health support has become widely known, we have not known what population is psychologically at a high risk, and which methods are effective for the residents' mental health in each phase has not been clear. Furthermore, how local governments operate a long-term mental health center depending on recovery phases is also unclear.

\section{The post-disaster mental health system in Japan}

Historically, two turning points contributed to the development of a post-disaster mental health system in Japan; the Hanshin-Awaji earthquake in 1995 (magnitude 7.3; 6,434 people died), and the Great East Japan Earthquake (GEJE) in 2011 (magnitude 9.0; 19,630 people died) [5]. The emergency mental health support system and the long-term mental health support system were established after these two major disasters.

An emergency mental health support system did not exist before the Hanshin-Awaji earthquake in 1995, and various groups formed ad-hoc support teams that provided services in the disaster-affected areas. These efforts were marred by unfortunate incidents where rescue teams inflicted damage on the survivors because they lacked the appropriate skills to carry out their operations or were confused owing to the prevailing circumstances [6]. Based on these experiences, mental health care teams consisting of psychiatrists, nurses, and social workers were formed and dispatched to the disaster-affected areas by 
the GEJE in 2011. The need for well-trained professionals who specialize in emergencies was recognized and the government of Japan through the Ministry of Health, Labor and Welfare set up a formal system that consisted of a Disaster Psychiatric Assistance Team in 2013 [7].

Local governments and public health centers play an important role in supporting communities in their long-term recovery following disasters in Japan. However, since these facilities could not sufficiently support communities when the scale of the disaster was overwhelming, the Japanese government and the affected municipalities established new organizations such as the Disaster Mental Health Care Centers (DMHCC), which specialized in reconstructing local mental health systems. For example, Hyogo DMHCC was established in response to the Hanshin-Awaji earthquake in 1995, and the Niigata DMHCC was established in response to the Niigata-Chuetsu earthquake in 2004 [8]. Similarly, three DMHCCs were established in the prefectures of Iwate, Miyagi, and Fukushima in the aftermath of the GEJE in 2011.

\section{The GEJE and Miyagi DMHCC}

On March 11, 2011, an earthquake and tsunami with a magnitude of 9.0 hit the eastern part of Japan, which left 19,630 dead, 6,230 injured. In addition, 2,569 people were reported as missing [5]. About 400,000 people were evacuated to emergency shelters right after the disaster. Prior to the disaster, the relatively rural areas affected by the earthquake did not have adequate mental health services. Since the Japanese government was forced to cope with the situation at hand in a relatively short period of time, the DMHCCs were established as community health recovery centers in the three prefectures of the Tohuku region.

The Miyagi DMHCC was established in December 2011, eight months after the disaster. It consisted of 40 staff members, divided among three branches. The team consisted of various mental health professionals: psychiatrists, psychiatric social workers, public health nurses, psychologists, nurses, and occupational therapists. The Miyagi DMHCC supported residents through home visits, telephone consultations, and counseling for walk-in visitors. These were some of the ways that the residents are connected to Miyagi DMHCC.

\section{Methods}

The study used data collected by the Miyagi DMHCC that included personal information of residents who were supported by the center from 2013 to 2018 . As this study was a retrospective chart review without considering personal information, no informed consent was obtained from any individual. We collected information about the age, sex, support methods, and consultation pathways from the database of Miyagi DMHCC. The cumulative number of individuals supported by the center in Miyagi prefecture was used to clarify the mental health system in Miyagi before the disaster and the function of Miyagi DMHCC. Although the Miyagi DMHCC started in 2012, the data in 2012 were incomplete and hence excluded. The individuals whose details of sex and age were incomplete were excluded from our analysis (45 entries were incomplete with respect to sex, whereas 1,394 entries were incomplete with respect to age). This study was approved by the Miyagi Disaster Mental Health Care Center Ethics Committee (Ethical Approval Reference No. 2020-03).

For the descriptive analysis, continuous variables were expressed as means (SD), and categorical variables were expressed as percentages. First, a chi-square test of independence was conducted between the sexes with regard to the annual number of individuals supported to confirm whether there is a sex difference in the number of supports. Second, a chi-square test of independence was conducted in the number of the support methods utilized per year to reveal the shift in support methods over time. Third, analysis of variance (ANOVA) was conducted by using the support years as an independent variable and the mean age as a dependent variable, and multiple comparisons using Tukey's HSD were performed for all categories in which the ANOVA revealed significant differences. Values of $p<0.05$ were referred to as statistically significant. Data were analyzed using SPSS (version 21.0; IBM SPSS, Armonk, NY).

\section{Results}

The major organizations that support residents with mental health problems are local municipalities and public health centers in Japan. Figure 1 shows the annual number of residents who needed mental health support from 2009 to 2017 . However, it did not include the number of residents who visited medical facilities. The residents who needed mental health support in Miyagi prefecture dramatically increased from 16,273 in 2011 to 29,766 in 2012 . The annual mean number of individuals supported by the DMHCC from 2012 to 2017 was 6,850.

Table 1 shows the age, sex, and the method of support given to the individual on a yearly basis. The cumulative number of individuals supported by the center from 2013 to 2018 was 39,680 (Men = 19,930 and Women = 19,750) and the annual mean number of individuals supported was 6,613. A chi-square test of independence demonstrated significant differences between the sexes with regard to the annual number of individuals supported by the center $\left[\mathrm{X}^{2}(5, \mathrm{~N}=\right.$ $39,680)=87.6, p<.001]$. According to the standardized residual analysis, the center supported a higher number of women in 2013 and a higher number of men in 2014.

The Miyagi DMHCC mainly supported residents by three major methods: home visits, counseling for walk-in visitors, and telephone counseling. A chi-square test of independence demonstrated significant differences in the number of the support methods utilized by patients by the years $\left[X^{2}(15, N=39,680)=1922.0\right.$, $p<.001]$. According to the standardized residual analysis, the number of home visits from 2013 to 2015 was frequent, and the number of counseling for walkin visitors was frequent in 2017 and 2018.

The overall mean age was 54.9 years $(S D=17.9)$, while the mean age of men was 54.3 years $(S D=17.1)$, and the mean age of women was 55.5 years $(S D=$ 18.6). There was a significant difference in the mean age between sex by using an independent-sample t-test $[\mathrm{t}(39,678)=6.44, \mathrm{p}<.001,95 \% \mathrm{Cl}(0.80-1.51)]$. The one-way between-subjects ANOVA also revealed significant differences in the annual mean age $[F(5,39,674)=144.1$, $p<.01]$. A post hoc Tukey HSD test showed that the mean age in 2017 and 2018 was significantly higher than in other years, and the mean age in 2013 was lower than that of the years between 2015 and $2018(p<.01)$. 
The clients were connected to the Miyagi DMHCC through various pathways. Since some clients were introduced via several organizations, the responses to the variable consultation pathway included more than 1 pathway. Thus, we did not assess the statistical significance of the difference. However, we observed that the ratio of residents who visit the center of their own accord increased continuously, whereas those who used the health survey pathway to connect to the Miyagi DMHCC decreased continuously.

\section{Discussion}

After the disaster, it was predictable that the demand for mental health services would increase because a vast number of residents experienced psychological trauma as a result of the disaster. Owing to a lack of mental health resources in the disaster-affected area in the aftermath of the 2011 earthquake the local municipalities and the public health centers found it challenging to treat all of the residents adequately. In response to this growing need for mental health services, the local administration of Miyagi Prefecture immediately decided to establish the DMHCC and collaborated with local municipalities to conduct a health survey of the affected residents who were financially supported by the Miyagi Prefecture [9] [10]. The Miyagi DMHCC collaborated with the local municipalities and confirmed the high-risk residents through home visits and provided them the appropriate support. Figure 1 shows that the number of residents who needed mental health support dramatically increased after the 2011 disaster, and the Miyagi DMHCC continuously followed up on many of the additional residents from 2012.

Over half of the support was by home visits from 2013 to 2015, and the number of home visits during those years was statistically higher than in other years. Many of these residents were identified through the health survey that was done by local municipalities. The mean ratio of the number of individuals who accessed the services of the DMHCC through the health survey to those who accessed the services through home visits of medical personnel or healthcare workers of Miyagi DMHCC was 0.52 from 2013 to 2015 [ $n=2,103(55.9 \%)$ in 2013, $n=2,124(50.0 \%)$ in 2014, and $n=2,449$ (55.9\%) in 2015]. The number of walk-in visitors was statistically lower from 2013 to 2015, and higher in 2017 and 2018 than in other years. From these results, it is clear that the post-disaster mental health care center should support residents through home visits in the first several years and subsequently set up a counseling system as residents start seeking support through their own volition. There were two reasons as to why residents sought mental health support after several years. First, the residents may have delayed seeking medical treatment and endured the painful situation for a long time, although they may have faced psychological distress. Second, it could also be the case that the psychological problems endured by the residents occurred later owing to other difficulties such as financial problems.

There were some noticeable results for sex. These might be based on the structure of society such as the labor situation in Japan: the employment rate of women is low and many men are responsible for their household finances. In the first three years, many of our activities tended to be limited to weekdays during the day, and the primary method we used was home visits. The residents with whom we come into contact with were more likely to be those at home during the day on weekdays. Inevitably, there was a possibility that the number of women was large in 2013. However, the number of men was high in 2014. According to age group and sex, the number of men aged between 40 and 50 years in 2014 was large [ $=723(10.1 \%)$, and many of these people had financial problems with unemployment. There is a possibility that men struggled to reconstruct their life, lost their job, and started seeking mental health support several years later.

The overall mean age of those seeking mental health services of individuals was the lowest in 2018, and that of both sexes in 2018 was also lower than in other years. There was a tendency that the mean age of individuals seeking help decreased over time. There was a possibility that elderly residents were predominantly likely to be found as psychologically high risk by the health survey and provided support in the first several years following the disaster, whereas younger residents were more likely to start seeking support of their own volition several years later. Evidence from a previous health survey indicates that many elderly individuals live alone in temporary housing, and their isolated existence and lifestyle are major issues that will have to be tackled [11]. These results indicate that public health officials will have to pay attention to the isolation suffered by the elderly following a large disaster. From the result of our study, we can make several suggestions to prevent isolation of elderly individuals. First, temporary housing (such as the temporary container housing built post disaster by local governments) should be built considering the accommodation of a mix of age groups in order to promote mutual aid between the residents. If many of the residents are elderly people in the temporary housing, it is more challenging to create a supportive environment. The structure through which the residents of all ages support each other is a vital concept for temporary communities. Second, local governments should regularly aim to understand the needs of elderly people who have the possibility of being isolated in their communities, and a long-term mental health care center should keep in touch with such elderly people after disasters. Therefore, staff in long-term mental health care centers must have group (community) management as well as outreach skills.

\section{Conclusions}

The number of residents who needed mental health support increased after the 2011 disaster. The Miyagi DMHCC was established in response to the disaster and supported residents who experienced psychological distress through several methods. It delivered psychological support to the elderly through home visits in the years after the disaster and younger people who sought the help of their own volition several years later. Therefore, the long-term mental health care center like the Miyagi DMHCC needs mental health workers who have community outreach skills, counseling skills, and a knowledge of techniques and strategies that prevent isolation.

It is clear that as post-disaster reconstruction efforts change phases, community residents faced diverse issues and needed mental health support. As an organization of supporters, we believe that it is necessary to quietly observe these changes and provide appropriate support at the correct time. Further research with regard to the increase in specific mental illnesses and the budget allocation for mental health is needed.

\section{Limitations}


This article has several limitations. First, we only analyzed the cumulative total number of patients supported, and the data we presented relied on statistics. Second, the individuals that sought medical help were part of the group of individuals who needed help. We could not analyze those residents who were supported by other organizations. Third, because the Miyagi DMHCC is not a medical organization, it mainly connected the residents with pre-existing community resources. As a result, we are unable to ensure that all individuals seeking support recovered, and we could not analyze the extent to which our efforts contributed to an individual's recovery. Finally, the residents on whom long-term mental health care centers should focus may be different depending on the structure of the communities before disasters. The problems of the communities may be different from those explored in this study if the main industry of the region is more urbanized, the residents are younger, and the employment rate of women is higher. The characteristics of Japanese society, the aging population, and the low female employment rate might have affected our results.

\section{Abbreviations}

ANOVA

analysis of variance

DMHCC

Disaster Mental Health Care Center

GEJE

Great East Japan Earthquake and Tsunami

\section{Declarations}

\section{Ethics approval and consent to participate}

This study was approved by the Miyagi Disaster Mental Health Care Center Ethics Committee (Approval No 2020-03).

\section{Availability of data and materials}

The datasets generated during and/or analyzed during the current study are available from the corresponding author on reasonable request.

\section{Competing interests}

The authors declare that they have no competing interest.

\section{Funding}

The authors declare no conflicts of interest associated with this manuscript.

\section{Authors' contributions}

NF designed the project and finalized the manuscript. SC conducted the data analysis and assisted with drafting the manuscript. All authors read and approved the final version of the manuscript for publication.

\section{Acknowledgments}

We thank all the participants who contributed to this study. We also would like to acknowledge the following individuals: Dr. Yoshiharu Kim (National Center of Neurology and Psychiatry) and Dr. Masaharu Maeda (Fukushima Medical University).

\section{References}

1. McFarlane AC, Williams R. Mental health services required after disasters: Learning from the lasting effects of disasters. Depress Res Treat. 2012;2012:970194.

2. HoustonJB, Spialek ML, Stevens J, First J, Mieseler VL, Pfefferbaum B. 2011 Joplin, Missouri tornado experience, mental health reactions, and service utilization: Cross-sectional assessments at approximately 6 months and 2.5 years post-event. PLoS Curr. 2015;7:dis.18ca227647291525ce3415bec1406aa5.

3. BrownLM, Framingham JL, Frahm KA, Wolf LD. Crisis counselors' perceptions and assessment of suicidal behavior among hurricane survivors receiving crisis counseling services. Disaster Med Public Health Prep. 2015;9(3):291-300.

4. North CS, Pfefferbaum B. (2013) Mental health response to community disasters: a systematic review. JAMA. 2013;310(5):507-518.

5. The damage situation caused by the Great East Japan Earthquake in 2011. Fire and Disaster Management Agency. 2020. https://www.fdma.go.jp/disaster/higashinihon/items/159.pdf. Accessed 24 November 2020.

6. Shinfuku N. Disaster mental health: lessons learned from the Hanshin Awaji earthquake. World Psychiatry. 2002;1(3):158-159.

7. Outline of the DPAT. Disaster Psychiatric Assistance Team. 2013. https://www.dpat.jp/images/dpat_documents/2.pdf. Accessed 24 November 2020.

8. H. Posttraumatic symptoms among victims of the Great Hanshin-Awaji Earthquake in Japan. Psychiatry and Clinical Neurosciences. 1998; 52(S1): S18S24. 
9. Matsuyama Y, Aida J, Hase A, Sato Y, Koyama S, Tsuboya T, et al. Do community- and individual-level social relationships contribute to the mental health of disaster survivors?: A multilevel prospective study after the Great East Japan Earthquake. Soc Sci Med. 2016,151:187-95.

10. Kusama T, Aida J, Sugiyama K, Matsuyama Y, Koyama S, Sato Y, et al. Does the type of temporary housing make a difference in social participation and health for evacuees of the Great East Japan Earthquake and Tsunami? A cross-sectional study. J Epidemiol. 2019;29(10):391-398.

11. Sato Y, Hashimoto A. How we should support victims according to types of residences after the Great East Japan Earthquake. Japanese Journal for Public Health Nursing. 2018;74(3):200-206 (in Japanese)

\section{Tables}

Table 1 Demographic characteristics of the residents who the Miyagi DMHCC supported

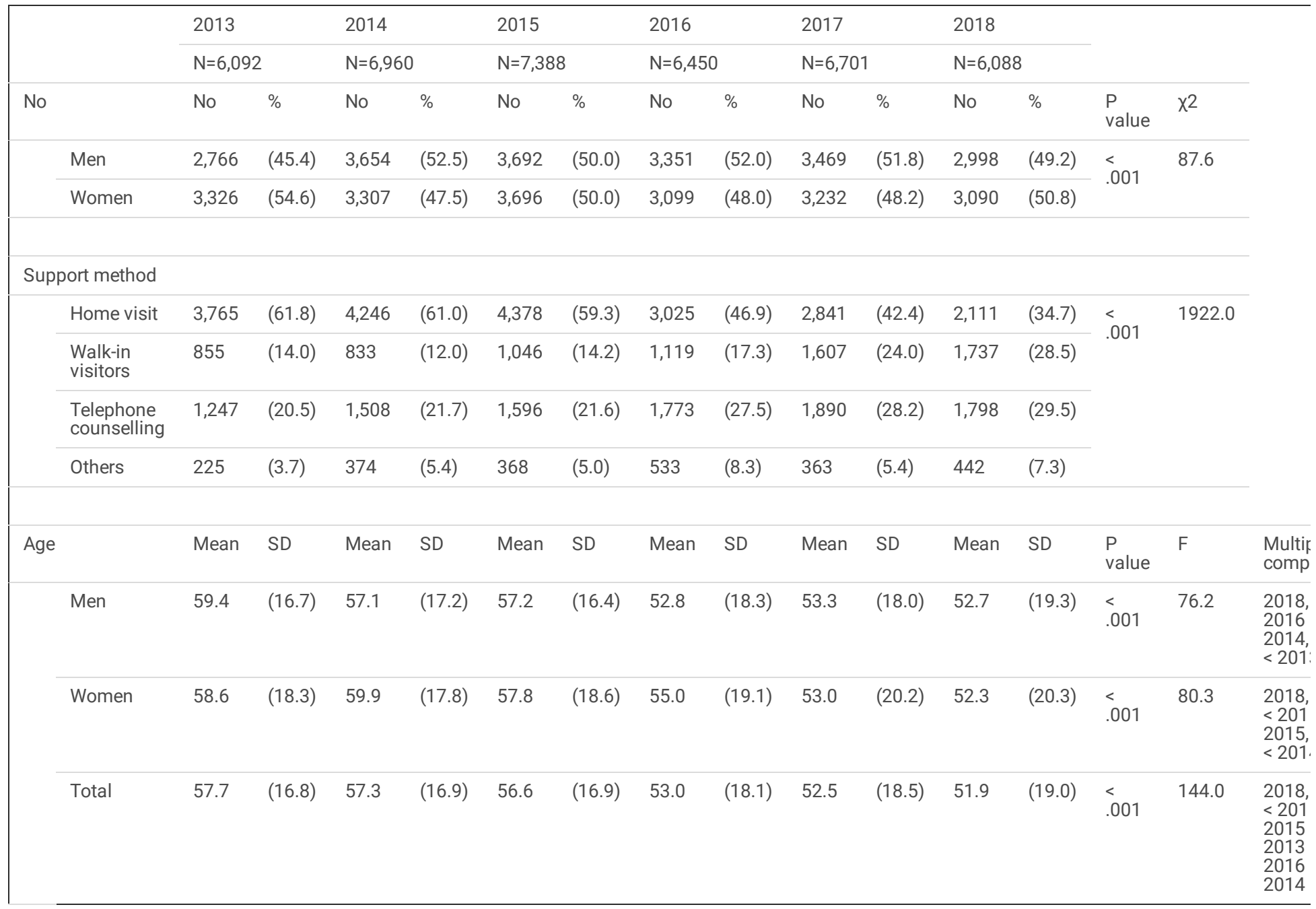

\section{Figures}




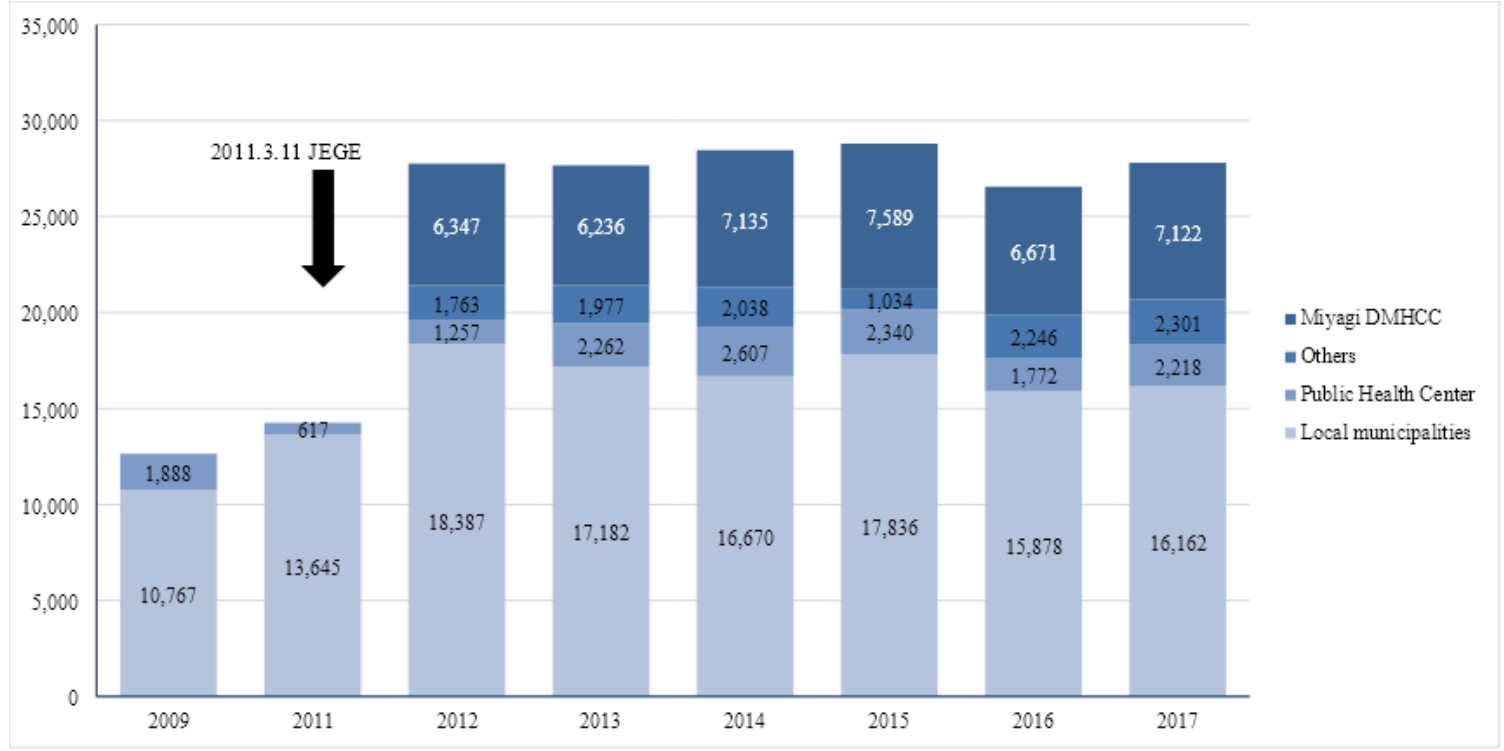

Figure 1

The cumulative number of mental health supports in Miyagi prefecture from 2009 to 2017. GEGE, Great East Japan Earthquake and Tsunami; DMHCC, Disaster Mental Health Care Center.

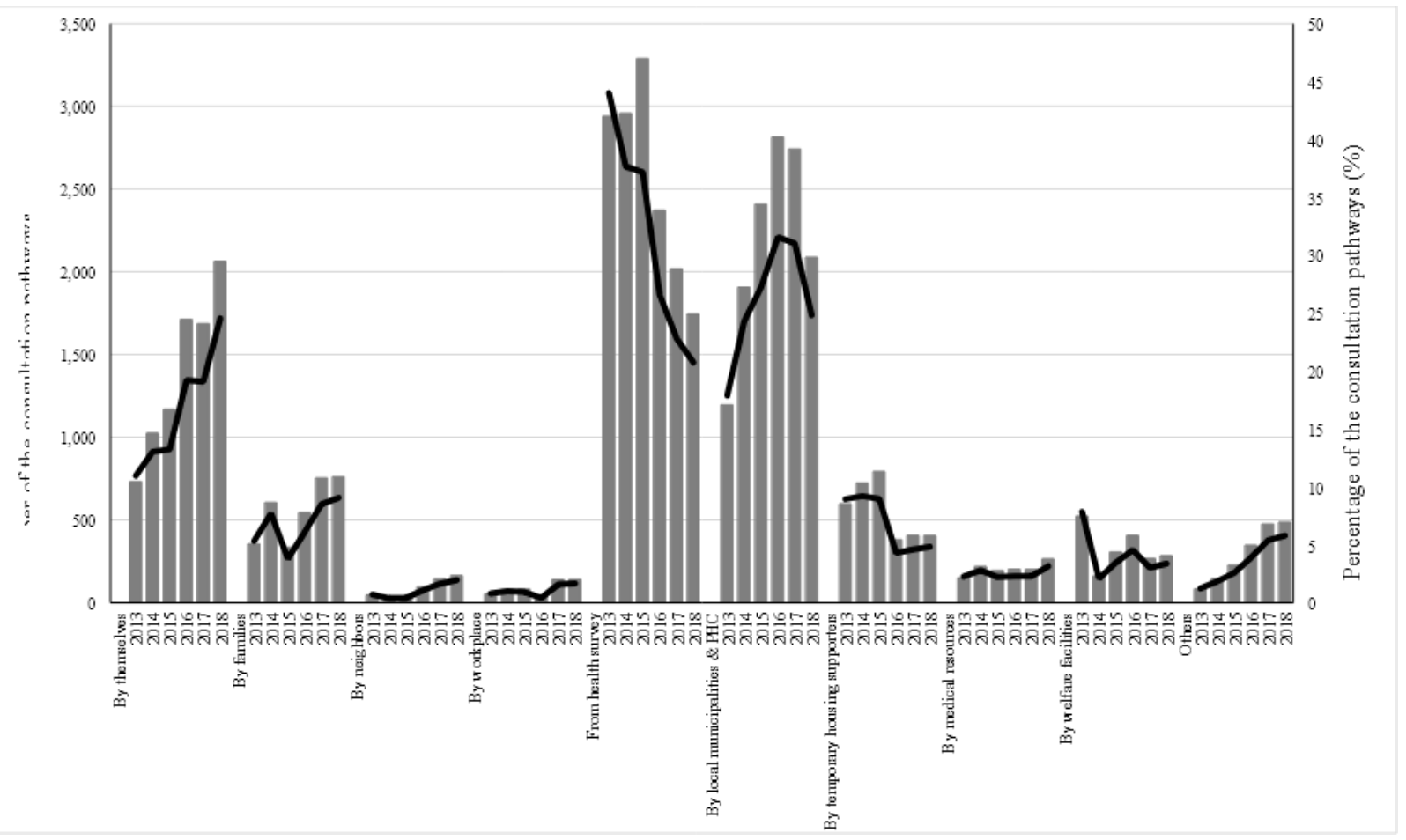

\section{Figure 2}

The number and the percentage of the consultation pathways. PHC, Public Health Center. 